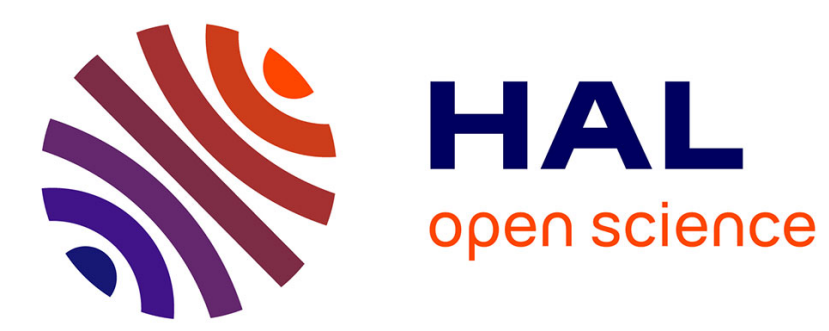

\title{
STRUCTURES BUILT BY AMPHIPHILES AND FRUSTRATED FLUID FILMS
}

\author{
J. Charvolin, J. Sadoc
}

\section{To cite this version:}

J. Charvolin, J. Sadoc. STRUCTURES BUILT BY AMPHIPHILES AND FRUSTRATED FLUID FILMS. Journal de Physique Colloques, 1990, 51 (C7), pp.C7-83-C7-96. 10.1051/jphyscol:1990707 . jpa-00231106

\section{HAL Id: jpa-00231106 https://hal.science/jpa-00231106}

Submitted on 1 Jan 1990

HAL is a multi-disciplinary open access archive for the deposit and dissemination of scientific research documents, whether they are published or not. The documents may come from teaching and research institutions in France or abroad, or from public or private research centers.
L'archive ouverte pluridisciplinaire HAL, est destinée au dépôt et à la diffusion de documents scientifiques de niveau recherche, publiés ou non, émanant des établissements d'enseignement et de recherche français ou étrangers, des laboratoires publics ou privés. 
STRUCTURES BUILT BY AMPHIPHILES AND FRUSTRATED FLUID FILMS

\author{
J. CHARVOLIN (1) and J.F. SADOC \\ Laboratoire de Physique des Solides, Bât. 510, Universite Paris-Sud, \\ F-91405 Orsay. France
}

\begin{abstract}
Résumé - Les interfaces entre deux liquides non miscibles, tels que $1^{\prime}$ eau et 1 'huile, peuvent être stabilisées par des molécules amphiphiles, telles que celles des savons, détergents et lipides. Les films interfaciaux construits par ces molécules présentent un polymorphisme remarquable : cristaux liquides ordonnés, structures "gonflées", solutions micellaires et micrómulsions désordonnées, liposomes et vésicules. Nous décrivons ces structures, en insistant sur celles des cristaux liquides, et nous en proposons une approche géométrique, qui conduit à les voir comme des structures de défauts et permet de développer la cristallographie des objets bi-dimensionnels nécessaire pour leur analyse.

Abstract - Interfaces between two non miscible liquids, such as water and oil, can be stabilized by amphiphilic molecules, such as soaps, detergents or 1ipids. The interfacial films formed by these molecules are known to build structures of an extraordinary polymorphism: ordered liquid crystals and "swollen" structures, disordered micellar solutions and microemulsions, liposomes and vesicles. We describe these structures, particularly the liquid crystalline ones, and propose a geometrical approach, which leads to consider them as structures of defects and is the basis of the crystallography of bi-dimensional objects necessary for their analysis.
\end{abstract}

\title{
1 - INTRODUCTION
}

Interfaces between two non-miscible liquids can be stabilized by molecules presenting an amphiphilic character, i.e. molecules which are assemblies of two chemical groups, each having a strong affinity for one of the two liquids. The best known example is that of the stabilization of water/oil interfaces by soaps, detergents or lipids. The interfacial films built by these molecules have liquid-like properties along their surface but, because of the structures of the amphiphilic molecules, 1iquid-crystalline curvature elasticities. Such films can be organized in structures of an extraordinary polymorphism. They are the cubic, cylindrical and lamellar liquid-crystalline structures with translational order along 3,2 or 1 dimensions, the nematic structures with orientational order only, the disordered micellar solutions and microemulsions, the liposomes and vesicles. Because of the very nature of the structural elements, 2-D fluid films with curvature elasticities and not $0-D$ solid atoms or molecules, this polymorphism can not be understood on the same bases than that of classical atomic or molecular organizations.

This lecture introduces the basic elements for describing and understanding the structures built by amphiphilic molecules in presence of one or two solvents. It might be seen, in its broadlines, as an introduction to the crystallography of systems of fluid films. The elements needed to analyse the behaviors and properties of these films, elasticities and statistical mechanics should be presented in W. Helfrich's lecture.

\section{BUILDING OF FILMS BY AMPHIPHILES}

\subsection{Amphiphiles at 1iquid/Iiquid interfaces}

It is a well known fact that polar and organic liquids are not miscible. For instance a dispersion of oil droplets in water, obtained by mechanical agitation, is not stable. It has too large an interfacial area. The system evolves, through the coalescence of its 
droplets, towards a stable state where the two liquids are separated by one flat interface of smallest area. However, if particular molecules, such as soaps, detergents, 1ipids and their mixtures with others such as alcohols, are added to the system, even at low concentrations, dispersions with quite large interfacial area may be stabilized. A much discussed example of such a stabilization today is that of microemulsions but, as we shall show very soon, several other structures are possible too.

The action of these molecules is easy to understand from their chemical structures. From the examples shown in $\mathrm{fig}$. 1 . it can be seen that they are built in two parts, one or two paraffinic chains attached to a polar group, which have different affinities for solvents.

$$
\begin{aligned}
& \text { a) } \mathrm{CH}_{3}-\left(\mathrm{CH}_{2}\right)_{\pi}-\mathrm{N}\left(\mathrm{CH}_{3}\right)_{3} \mathrm{Cl}
\end{aligned}
$$

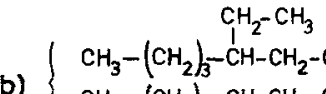

$$
\begin{aligned}
& \text { b) }\left\{\begin{array}{l}
\mathrm{CH}_{3}-\left(\mathrm{CH}_{2}\right)_{3}-\mathrm{CH}_{1}-\mathrm{CH}_{2}-\mathrm{CO}_{2}-\mathrm{CH}_{2} \\
\mathrm{CH}_{3}-\mathrm{CH}-\mathrm{CH}_{2}-\mathrm{CO}_{2}-\mathrm{CH}-\mathrm{SO}_{3} \mathrm{Na}
\end{array}\right. \\
& \mathrm{CH}_{2} \mathrm{CH}_{3} \\
& \text { c) }\left\{\begin{array}{l}
\mathrm{CH}_{3}-\left(\mathrm{CH}_{2}\right)_{15}-\mathrm{CO}_{2}-\mathrm{CH}_{3} \\
\mathrm{CH}_{3}-\left(\mathrm{CH}_{2}\right)_{15}-\mathrm{CO}_{2}-\mathrm{CH} \\
\quad \mathrm{CH}_{2}-\mathrm{PO}_{4}^{-}-\mathrm{CH}_{2}-\mathrm{N}^{+}-\left(\mathrm{CH}_{3}\right)_{3}
\end{array}\right. \\
& \text { d) } \mathrm{CH}_{3}-\left(\mathrm{CH}_{2}\right)_{9}-\mathrm{SO}_{3} \mathrm{Na} \\
& \text { e) } \mathrm{CH}_{3}-\left(\mathrm{CH}_{2}\right)_{8}-\mathrm{CH}_{2} \mathrm{OH} \\
& \text { f) } \mathrm{CH}_{3}-\left(\mathrm{CH}_{2}\right)_{15}
\end{aligned}
$$

Fig. 1 - Some examples of amphiphilic molecules: a) dodecyl trimethyl ammonium chloride (DTAC1), b) aerosol OT, c) a lipid, dipalmitoyl phosphatidine choline (DPPC), d) sodium sulfate (SdS), e) decanol, f) cetyl pyridinium chloride (CPC1).

The paraffinic chains have a good affinity for organic solvents, such as oil, but not for polar solvents, such as water. It is the opposite for the polar group. Such molecules are said to be amphiphilic. When put in presence of the solvents they can therefore build 2-D interfaces of the type of those shown in fig. 2 .
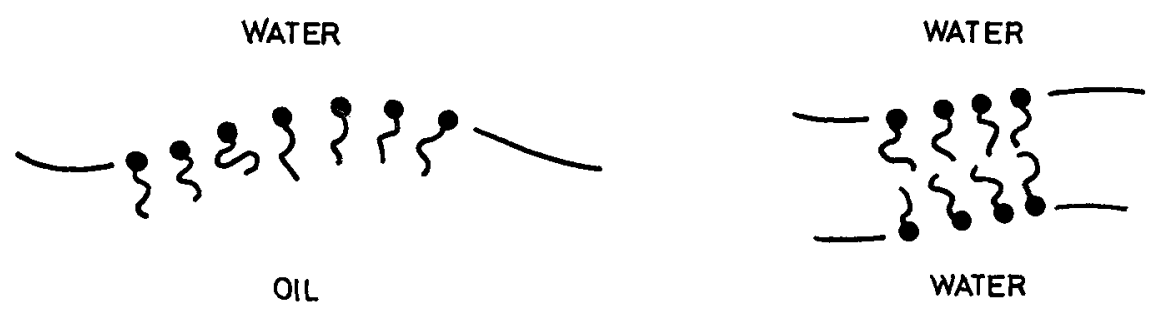

Fig. 2 - Interfacial films built by amphiphilic molecules: a) in presence of oil and water, b) in presence of water only.

The shapes and properties of these interfaces depend upon the structure of the molecules, the temperature and the concentrations, as shown by phase diagram studies. (The molecules shown above have a rather low molecular mass, a few hundreds, but copolymers with much larger mass, a few thousands, can exhibit amphiphilic behaviors also with different solvents). 


\subsection{Phase diagrams and structures}

Phase diagrams /1/ and structures /2,3/ have been extensively studied for years, mainly by optical observations of textures and small angle $X$-ray scattering. We present and discuss here a few typical examples only, limiting ourselves to the high temperature region of the phase diagrams where, as we shall demonstrate in the following paragraph, the behavior of the amphiphilic molecules at the interfaces is liquid-like.

The first of these examples is that provided by the binary mixture dodecyl trimethylammonium chloride/water shown in fig. 3. /4/

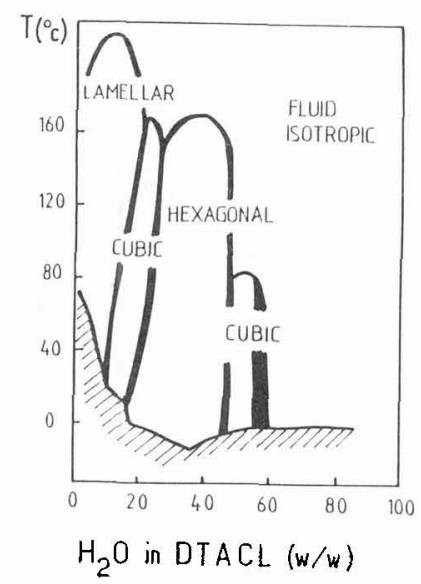

Fig. 3 - Phase diagram of the system DTAC1/water, from reference $/ 4 /$.

It displays the phases of most general occurence: lamellar ( $L \alpha)$, "bicontinuous" cubic ( $\left.Q^{\prime} \alpha\right)$, hexagonal $(H \alpha)$, "micellar" cubic $\left(Q^{\prime \prime} \alpha\right)$ and the micellar solution. The condensed notation used here is that proposed by $B$. Luzzati: the capital letter indicates the structure of the long range organization of the interfaces and the greek letter indicates the local state of the molecules which, in the cases considered here, is liquid-like $/ 2 /$. The classical description of the structures of the phases is given in fig. 4.

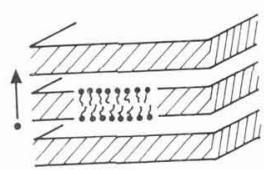

(a)

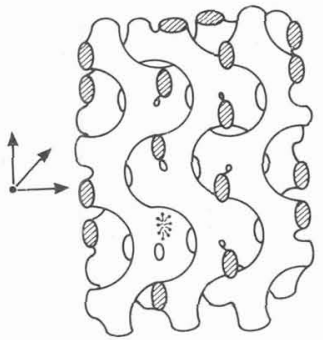

(b)

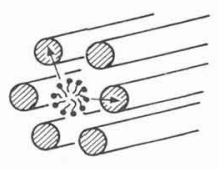

(c)

Fig. 4 - Schematic representations of the $L \alpha$ structure a), the $Q^{\prime} \alpha$ structure with space group $1 \mathrm{a} 3 \mathrm{~d} \mathrm{~b})$, the $\mathrm{H} \alpha$ structure $\mathrm{c}$ ). 
The lamellar structure is a periodic stacking along one dimension of bilayers of amphiphiles and layers of water. The "bicontinuous" cubic structure is a periodic entanglement along three dimensions of two labyrinths of amphiphiles separated by one film of water. The hexagonal structure is a periodic organization along two dimensions of cylinders of amphiphiles separated by a honeycomb-1ike film of water. The structure of the "micellar" cubic phase is not firmly established at the moment, it has been proposed to be built of either a labyrinth enclosing spherical micelles $/ 5 /$ of anisotropic micelles $/ 6 /$, all disposed on cubic lattices. We shall propose a third possibility in the fourth paragraph. In the micellar solution finite micelles are dispersed in water without any long range order; some systems are able to form giant cylindrical micelles whose behavior resembles that of 1 iving polymers /7/. The general trend in such phase diagrams is that, when the water content increases beyond that of the lamellar phase, water becomes the continuous medium and the interfacial curvature becomes more and more concave on the paraffinic side.

The second phase diagram is that of the aerosol OT/water mixture /8/. As can be seen in $f i g$. 5 it might be said to be a "mirror" image of the previous one.

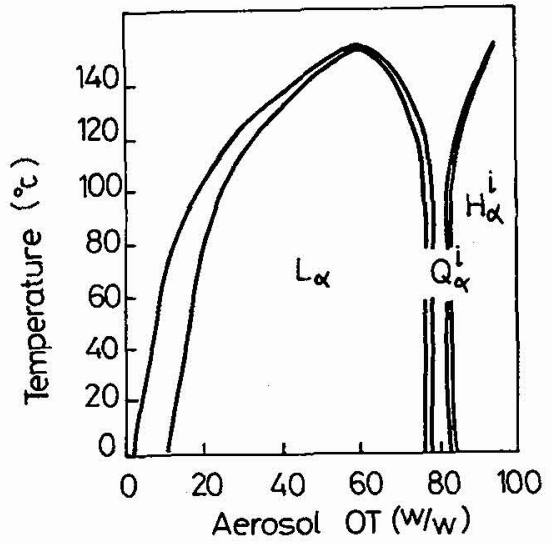

Fig. 5 - Phase diagram of the system aerosol OT/water, from reference $/ 8 /$.

The lamellar phase exists for high water content and the other phases appear for decreasing water contents. Moreover if the structures are similar, as far as the symmetries are concerned, their aqueous and paraffinic media are interchanged. For instance, the labyrinths of the "bicontinuous" cubic phase and the cylinders of the hexagonal phase are channels of water separated by a film of amphiphiles. This change is most obviously due to the structure of the molecule which, because of its two chains, forces the concavity of the interfaces in the other sense. The high water content region of the phase diagram is a phase separation zone containing a dispersion of lamellar domains in the isotropic solution. Lipids with two chains present this type of phase diagram also with, particularly, a much larger phase separation zone /9/. In some cases, observations by electron microscopy /10/ have shown that the lamellae of these dispersed domains are organised concentrically in onion-like structures called liposomes or multilamellar vesicles. From these liposomes it is possible to obtain, by various treatments presented in $/ 11 /$, unilamellar vesicles which are closed bags limited by one lamella only with diameter in the micron range, as shown in fig. 6 .

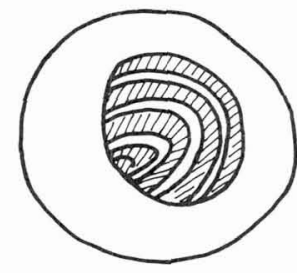

a)

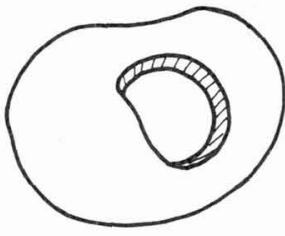

b)

Fig. 6 - Schematic representations of multilamellar a) and unilamellar b) vesicles. 
The addition of a third component, a second amphiphilic species, may induce interesting behaviors. Good examples are provided by soap/alcohol/water phase diagrams /1/. We do not develop this aspect here but just quote that new structures appear which are characterized by inhomogeneous interfacial curvatures associated to an inhomogeneous distribution of the two molecules within the aggregate $/ 12 /$ also, certain for a soap/alcohol ratio, it has been shown that the domain of the lamellar phase in these phase diagrams may extend up to very high water contents. Such "swollen" lamellar phase are obviously good candidates for studying forces between interfaces $/ 13,14 /$.

The last phase diagram considered here is that of the quaternary system sodium dodecylsulfate/ butanol/brine/toluene shown in fig. $7 / 15 /$.

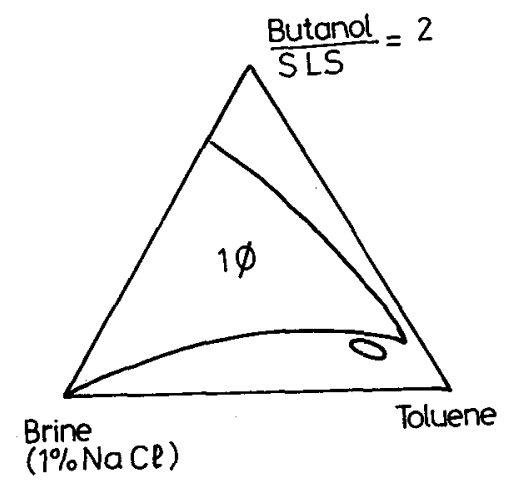

Fig. 7 - Pseudo ternary phase diagram of the SLS/butanol/toluene/brine system with its Iarge microemulsion domain.

The remarkable point of this phase diagram is the existence of a large monophasic domain extending from the water rich region to the oil rich region. This is the microemulsion domain where the structure changes continuously from droplets of oil in water, coated by the amphiphilic interface, to droplets of water in oil, coated by an interface of opposite curvature. The structural changes permitting the inversion of the interfacial curvature in the intermediate region are not clearly described and understood yet. Bicontinuous disordered structures of complex topology, with a labile interface of amphiphiles separating labyrinths of oil and water, have been proposed.

These phase diagrams exemplify the extreme versatility of the interfacial behavior. It is a function of many parameters related to the molecules, their concentrations and the temperature. In the next paragraph we shall try to see how far we can go in the understanding of this at the molecular level.

\section{MOLECULAR BEHAVIOR IN FILMS}

The extraordinary polymorphism of the films in the systems described above introduces the question of its relation with the molecular behavior. To tell it in an other way, does it exist a correspondence between the structures and the molecular conformations of the amphiphiles, particularly of their paraffinic chains?

This question was partly answered by X-ray scattering studies at large angles which showed that the paraffinic medium is disordered /2/. However it was necessary to develop other experiments, using techniques sensitive to local fluctuations, to describe this disorder in a more accurate manner. Among them NMR proved to be remarkably simple and efficient /16/. Relaxation studies showed first that the disorder is dynamical with similar characteristic times in all structures $/ 17,18,19 /$ : the chains are deformed by isomeric rotations around their $C \rightarrow C$ bonds, with correlation times of about $10^{-9-10^{-11}} \mathrm{sec}$, and diffuse along the interfaces, with translational diffusion coefficient of about $10^{-6}-10^{-7} \mathrm{~cm}^{2} / \mathrm{sec}$, as shown in fig. 8 . 


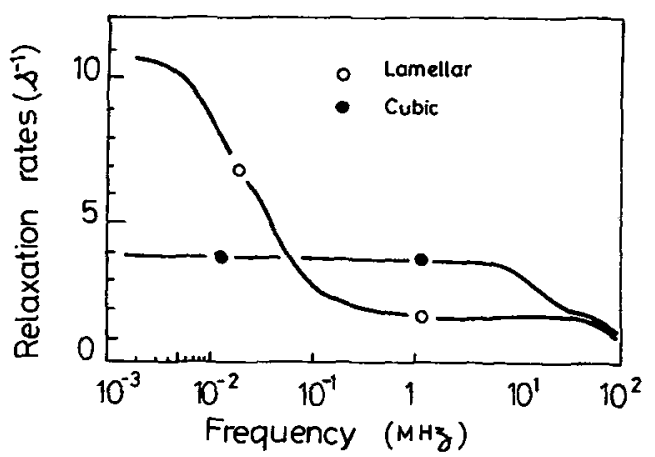

Fig. 8 - NMR relaxation curves, related to spectral densities, obtained in the lamellar and cubic phases of the potassium laurate/water system, the relaxation due to rapid deformations appears in the $10^{2}$ range, that due to the translational diffusion in the $10^{1}$ range and that due to very slow deformations or collective motions in the $10^{-2}$ range.

It is quite noticeable that these figures are close to those known for melted paraffins of similar chain length. Then the development of NMR studies of deuterated molecules $/ 20 /$ and of $\mathrm{C}^{33} \mathrm{NMR} / 28 /$ permitted to show that the amplitude of the deformations are about the same in all structures, ordered 1 iquid crystals $/ 16,21 /$ as well as in disordered micellar solutions $/ 18 /$, as shown in fig. 9 .

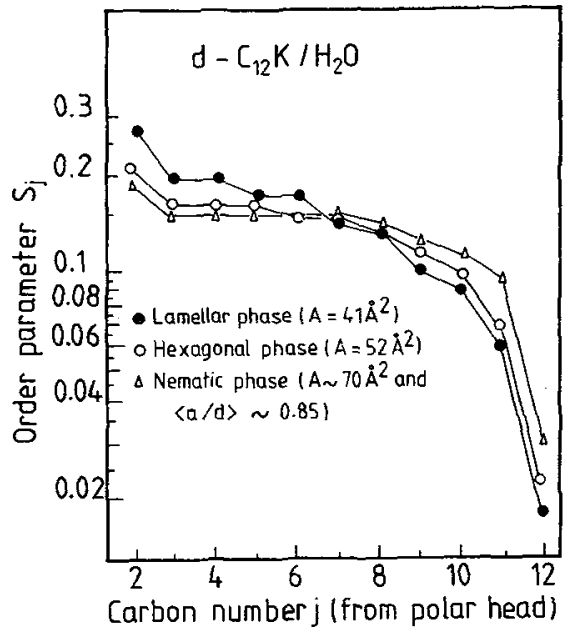

Fig. 9 - CD bond order parameter curves obtained in three phases of the potassium laurate/ water system, $A$ is the mean area per molecule at the interface and a/d the aspect ratio of the aggregates in the nematic phase.

Thus, there does not appear any definite correlation between one structure and the molecular behavior in it. The chains are about equally disordered in all structures, in spite of important changes of interfacial curvature and mean area per molecule at the interface.

The idea of analyzing ordered liquid crystalline structures in the same terms than those used to describe classical molecular crystals was therefore abandoned, as the molecule is not the element of structure. They can be seen as ordered entanglements of two disordered liquids separated by an interface, and their element of structure is the interface built by the molecules or better, to respect the basic symmetry, the film formed by two facing interfaces /22/. 


\section{PRINCIPLES OF THE ORGANISATION OF INTERACTING FILMS}

Coming back to the phase diagrams and structures described in the second paragraph, we drew the conclusion that similar structures appear along a similar sequence, whatever the details of the chemical structures of the molecules. For instance, in the phase diagram of $\mathrm{fig}$. 3 , the lamellar phase is followed by a "bicontinuous" cubic phase then by a hexagonal one and, finally, by a micellar phase when the water content increases. Similar sequences, or part of it, can be found in other diagrams too when their dominant parameter varies. These similitudes can not but hold to the common amphiphilic nature of the molecules and to their ability to build interfaces organized in symetric films, as shown in $\mathrm{fig} .10$.

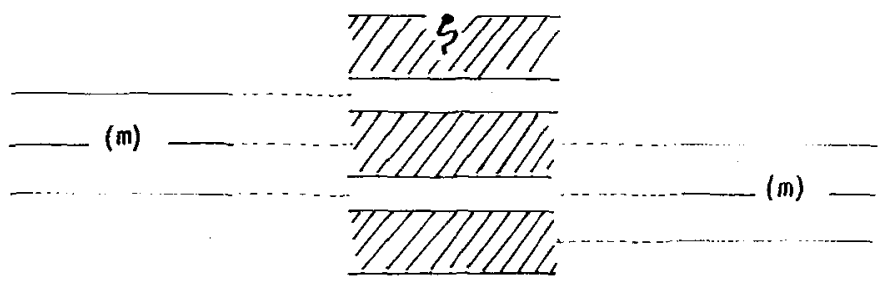

Fig. 10 - Definition of the film in a lamellar structure. It can be either a film of amphiphiles limited by two half layers of water with its middle surface in the bilayer, or a film of water limited by two monolayers of amphiphiles with its middle surface in the water layer.

The question we want to address now is that of the understanding of the structural polymorphism of films in interaction. Can it be described on a simple basis common to all systems?

To explain our point of view on this problem let us consider the classical methods which were developed to analyze organizations of atoms and molecules. It is well known that it is not possible to deduce their structures from their interactions potentials directly, in a simple manner. Two directions were taken. The first consists in calculating the free energies of interacting particles assembled on lattices of given symetries. The comparison of the results with the observed symetries is a test of the validity of particular potentials. In this case the catalog of lattices is provided by crystallography, which can be defined as the investigation of possible geometrical configurations to pack together particles of certain symmetries. The second direction is that of the simulation of the system of particles in a computer which build a structure. This is a very heavy method, particularly in the case of complex 3-D structures. We are now facing the same problem in the case of our systems, but at a higher level of complexity because we are dealing with $2-D$ objects, films, and not $0-D$ objects, atoms or molecules.

Up to now, the direction of simulation on a computer could not be followed, because of the high numbers of molecular degrees of freedom and molecules per unit cells. The first direction could not be strictly followed either, in the absence of any crystallographic information about film organizations. Nevertheless this information could be replaced by considering the structures as they are given by the observations, at least for the simplest of them, lamellar, hexagonal and micellar. Free energy calculations were developed on this basis with a precise account of the forces acting in the system, between molecules, ions, interfaces, particularly those of electrostatic origin /23/. A quite reasonable agreement was observed between the calculated locations of lamellar, hexagonal and micellar structures in some phase diagrams and the experimental data, particularly for ternary diagrams soap-alcohol-water. This gave a strong weight to electrostatic forces. However, recent observations of rectangular or nematic phases within domains of certain of these phase diagrams thought before to be hexagonal or micellar $/ 12 /$, and of intense hexagonal or rectangular fluctuations within their lamellar phases thought before to have infinite lamellae $/ 24 /$, should temper this first conclusion. In anyway, the general success of this approach pushed us to think of a real crystallographical basis to support it. As for atoms or molecules, the way films can be packed are obviously driven by their "shape", or their interfacial curvatures, and their distances; but, in the case of films, strong topological constraints appear which impose states of frustration. 


\subsection{Interplay of forces and frustration}

The forces acting in a system of films of amphiphiles are many of different origins $/ 25 /$. They are not all well known at the moment. However their detailed knowledge is not necessary at this stage. We just need to make use of the fact that the role of their components normal to the interfaces is to maintain constant distances between them, if the interfaces are supposed homogeneous, and that the role of their components parallel to the interfaces is to determine the interfacial curvatures, as they do not vary necessarily in concordance at different levels within the layers limiting the film. Owing to the symmetry of the film with respect to its middle surface it is clear that the fact that two facing interfaces may have symmetric curvatures is not always compatible with constant distances between the interfaces, if a lamellar-type stacking is kept, as shown in fig. 11.
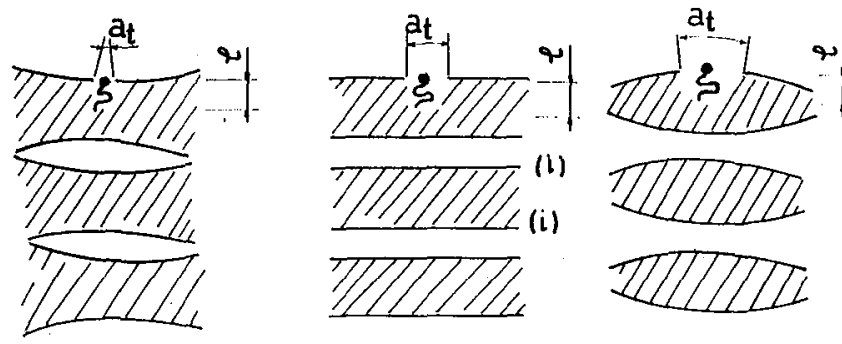

Fig. 11 - Schematic representation of a periodic system of films with flat interfaces (center): constant interfacial distances and zero curvature are compatible in $R_{3}$. The same with symetrically curved interfaces (left and right): constant interfacial distances and non zero curvatures are no longer compatible in $R_{3}$ and the system becomes frustrated.

The left and right drawings of this figure present situations of conflict between forces normal to the interfaces and forces parallel to them. Such a situation is a typical case of frustration. The forces are therefore obliged to compromise and new structures should be built. We now look for the possible geometrical configurations of the films optimizing this frustration.

\subsection{Search for solutions}

A frustration is directly related to the structure of the space containing the system under study. As shown in $\mathrm{fig}$. 11, where a system embedded in the usual flat 3-D Euclidean space $\mathrm{R}_{3}$ is represented, the situation with flat interfaces is the only situation compatible with constant interfacial distances in this space. However, if the system is embedded in an adequately curved space, the situations with curved interfaces may become compatible with constant interfacial distances. (This is formally similar to problems of bi-dimensional tilings with regular pogygons and tri-dimensional packing of regular polyhedra /26/). Indeed ideal lamellar-1ike structures without frustration, conciliating the two antagonistic constraints, can be built in curved spaces. Such ideal structures have of course no reality but are useful, as starting points, to generate possible configurations in the flat Euclidean 3-D space. For this it is necessary to map the curved space onto the flat space. A classical example of such a process with 2-D spaces is that of the mapping of a sphere onto a plane. A sphere can not be deformed into a plane without being torn, except if matter is added during the process. If a structure is present on the sphere the introduction of matter must respect the symmetxy of this structure. This is generally known as the Volterra process in condensed matter physics, and corresponds to the creation of discontinuities, or defects, in the structure. Such defects can be defects of translation, or dislocations, and rotation, or disclinations $/ 27 /$. As the direct displacements on a sphere are rotations the defects needed to map it onto a flat space are disclinations only. In a similar way, the mapping of the 3-D curved space, containing the lamellar-1ike ideal structure, onto the $3-D$ flat space, where the real structure should be found, is obtained by introducing a network of disclination lines. This network must respect the symmetries of the structure without frustration in the curved space and its density of disclination is determined by the curvature of the curved space. The possible configurations obtained that way can therefore be seen as structures of disclinations. The application of this method to frustrated films can be found in a series of recent articles /28-32/. In this article we give a brief sketch of it in the case of the cubic phases only, before giving a brief summary of the general results. 


\section{3. "Bicontinuous" cubic structures $Q^{\prime} \alpha / 29,32 /$}

In this case the whole periodic system of frustrated fluid films can be represented without frustration in $S_{3}$ by putting the middle surface of the film onto the spherical torus $T_{2}$. This toroĩdal surface separates $S_{3}$ in two identical subspaces and admits a $\{44\}$ regular tiling. Disclinations are to be introduced around the symmetry axes of the relaxed structure in a manner respecting its symmetry. As shown in fig. 12 half a torus has to be indroduced in between the lips of a cut limited by a $C_{2}$ axis normal to the surface of the torus.

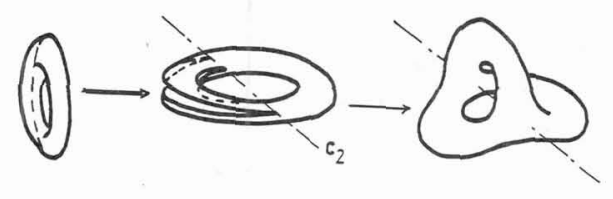

Fig. $12-\mathrm{A}-\pi$ disc1ination around a $\mathrm{C}_{2}$ axis of $\mathrm{T}_{2}$ in $\mathrm{S}_{3}$. A cut is made in the torus, the 1 ips of the cut are separated (middle), half a torus (left) is inserted in between the 1ips, a torus of higher genus is generated (right).

This is a a- $\pi$ disclination which does not affect the property of the surface of partioning the space in two identical sub-spaces. This process leads to a configuration which has the bicontinuous topology of cubic phase $Q^{\prime} \alpha$. In the course of this process the squares of the $\{4,4\}$ tiling of the spherical torus are transformed into hexagons without any modification of the nupber of polygons per vertex, or the $\pi / 2$ values of the angles, as shown in $f i g$. 13 .

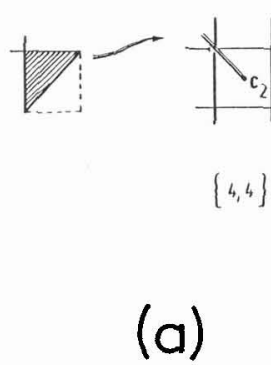

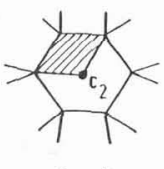

$\{6,4\}$

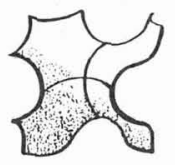

(c)

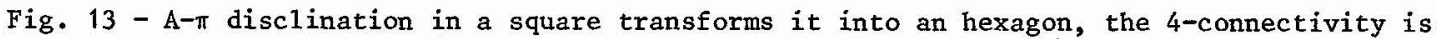
preserved at every vertex, if the original square is in a plane with zero Gaussian curvature the plane transforms into a hyperbolic surface with negative Gaussian curvature.

A new surface is generated by this disclination process, it admits a $\{6,4\}$ tiling. Obviously such a tiling is not Euclidean, or planar, and the new surface has a constant negative Gaussian curvature, it is a hyperbolic plane. A hyperbolic plane, a bi-dimensional space with constant negative Gaussian curvature, can not be embedded in $R_{3}$ without metric distorsions: However, the properties of such hyperbolic planes can be studied using the Poincaré's model in the Euclidean plane. A hyperbolic plane with a $\{6,4\}$ tiling is represented in fig. 14a together with its orthoscheme triangles or basic assymetryc units. These triangles have angles of $\pi / 2$, $\pi / 4$ and $\pi / 6$ and are not Euclidean. The analysis of their possible configurations, straight or curved sides, informs about the possible surfaces in $\mathrm{R}_{3}$. 


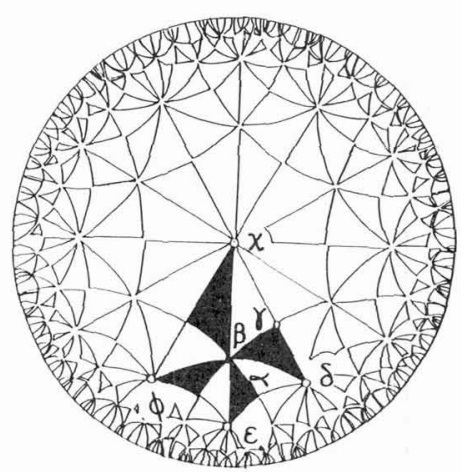

(a)

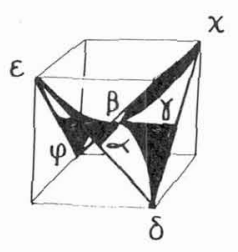

(b)

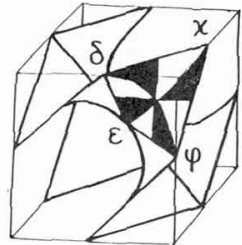

(c)

Fig. 14 - Poincaré's representation of a hyperbolic plane with $\{6,4\}$ tiling and its orthoscheme triangles with angles $\pi / 2, \pi / 4, \pi / 6$ (a), the organization of quadrangles $(\alpha, \beta, \gamma, \delta)$ in the saddle $(\delta, \varepsilon, \phi, \chi)$ in $R_{3}(b)$, the monkey saddle cel1 with a set of 6 quadrangles $(\delta, \varepsilon, \phi, \chi)$ (c).

We consider only one case here, when the two sides of the right angle are straight, other cases are discussed in $/ 29 /$. These straight sides build a lattice of intersecting straiht 1 ines whose element is a non planar quadrangle $(\alpha, \beta, \gamma, \delta)$ with three angles of $\pi / 2$ and one of $\pi / 3$. These elements can be assembled four by four in a more symetrical skew quadrangle $(\delta, \varepsilon, \phi, X)$ with four angles of $\pi / 3$ and four equal sides, fig. 14b. These quadrangles are regularly organized on the hyperbolic plane and it was shown by Schoenflies and Schwarz that, when embedded in $R_{3}$, they build a cubic lattice whose translation cell is shown in fig. 14c. Schwarz has shown that this cubic lattice is the support of an infinite periodic minimal surface (IPMS), called $F$ or $D$, separating $R_{3}$ ir two identical sub-spaces whose labyrinths are the two interwoven, but not connected, lattices of rods shown in $\mathrm{fig} .15$.

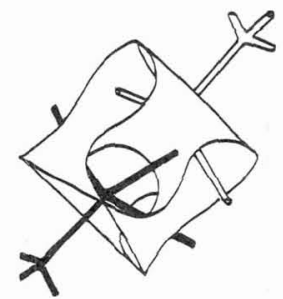

(a)

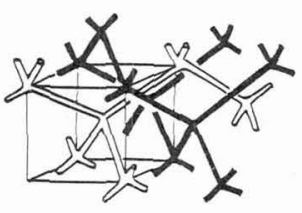

(b)

Fig. 15 - The translation cell for the F surface of Schwarz and the labyrinths of the Pn3m cubic structure separated by this surface.

These two interwoven labyrinths are indeed identical to those which were determined for a cubic structure with space group $\mathrm{Pn} 3 \mathrm{~m} / 5 /$.

The two other configurations possible for the orthoscheme triangle, one with a straight hypotenuse and another with all sides curved, permit to build two other cubic phases /5/: one with Im3m symnetry, where Schwarz P surface separates labyrinths of rods connected 6 by 6 , another with Ia3d symetry where Schoen's $G$ surface separates labyrinths of rods connected 3 by 3 , see alfo fig. $4 / 33 \%$. (A recent analysis of translation sub-groups in the $\{6,4\}$ hyperbolic plane $/ 32 /$ has confirmed this result, and is presented at the conference by J.F. Sadoc) 


\section{4. "Micellar" cubic structures $Q " \alpha / 30 /$}

In that case the whole periodic system of frustrated fluid films can be represented without frustration in $S_{3}$ by putting the middle surface of the film onto the "equatorial" sphere $S_{2}$ of $\mathrm{S}_{3}$. The relaxed structure admits $\mathrm{C}_{2}$ symmetry axes which are great circles of $\mathrm{S}_{2}$. The introduction of a disclination along one of these axes brings in a third finite sub-space, as shown in fig. 16 .

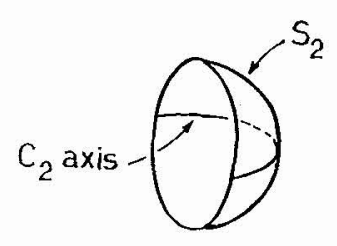

(a)

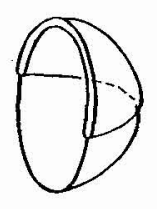

(b)

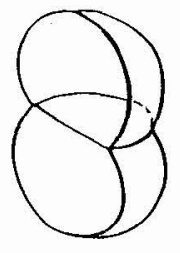

(C)

Fig. 16 - A disclination along a $C 2$ axis of S2 in S3 (for the sake of clarity only half a sphere S2 is shown in this stereographic projection of S3 onto R3, and the symmetric film supported by $\mathrm{S} 2$ is not represented):

a) S2 separates $S 3$ in two identical sub-spaces,

b) S2 is partly split in two sheets limited by the C2 axis,

c) a third sub-space, identical to the two first, is introduced between the sheets.

The repetition of this process, up to the point where the curvature of $s_{3}$ has decreased to zero, just multiplies the number of identical finite sub-spaces. We get therefore a spacefilling packing of identical finite cells. The law of topological stability imposes the search for eventual space filling assemblies of regular polyhedra, or polytopes, having three faces per edge and four edges per vertex or, having three faces belonging to the same polyhedron around one vertex and three polyhedra around one edge /34/. This means the polytopes of the $\{\mathrm{p}, 3,3\}$ family. They are four which, unfortunately, exist in curved spaces on $1 \mathrm{y}$ :

- in spherical spaces with constant positive Gaussian curvatures $\{3,3,3\},\{4,3,3\}$ and $\{5,3,3\}$

- in a hyperbolic space with constant negative curvature $\{6,3,3\}$.

As there is no $\{p, 3,3\}$ polytope in a flat Euclidean space our problem admits no solution with identical regularpolyhedral cells. We are therefore driven to search for eventual non-regular but, nevertheless, periodic solutions. For this it is useful to consider the fact that the regular $\{p, 3,3\}$ polytopes are found in spaces of decreasing curvatures and are therefore met one after the other during the progressive introduction of disclinations needed to map $S_{3}$ onto $R_{3}$. The polytope of interest is obviously $\{5,3,3\}$ which exists in the space of lowest positive curvature. We must start from it and add in it new disclinations realizing the final mapping of $S_{3}$ onto $R_{3}$. As the first disclinations are contained in the surfaces, the new ones can not but be normal to them. Indeed it is known that disclinations normal to pentagonal faces of a dodecahedron transform it into the tetrakaidecahedron and hexakaidecahedron shown in $\mathrm{fig}$. 17 .

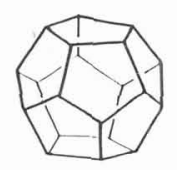

(a)

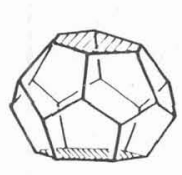

(b)

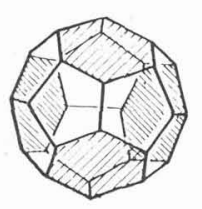

(c)

Fig. 17 - Transformation of a dodecahedron (a) into a tetrakaidecahedron (b) by one - $2 \pi / 5$ disclination along an axis normal to pentagonal faces and into a hexakaidecahedron (c) by four hàlf disclinations. 
These non-regular polyhedra are particularly interesting here as it is known that, when slightly distorted, they can be packed so that they build periodic space-fillig assemblies of cells. These organizations should be those of the film and micelles permitted by the properties of our Euclidean space, the film being supported by the faces of the polyhedra and each cage delimited by it containing a micelle. Several structures can be built along the above principle and, among them, we can distinguish two large families according to the fact that their dihedral and edge angles stay close to those of fluid films balancing their tensions or not. If we limit ourselves to the first case we are left with two relatively simple structures: one has space group Pm $3 n$ and its crystallographic unit cell contain 2 dodecahedra and 6 tetrakaidecahedra, the local arrangement of its polyhedra being shown in fig. 18 .

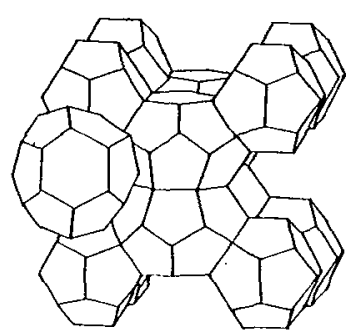

(a)

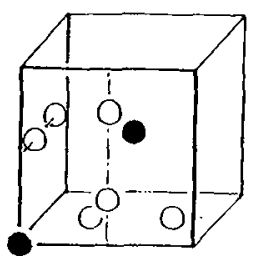

(b)

Fig. 18-Aggregation of slightly distorted 12-hedra and 14-hedra in a periodic structure, on the right the positions of the 12-hedra (o) and 14-hedra (o) in the cell of the Pm3n lattice.

The second has space group Fd3m and its unit cell contains 16 dodecahedra and 8 hexakaidecahedra.

\subsection{General results}

The first result is that there are three disclination processes possible giving access to three topological classes /28/. One corresponds to structures where a unique film separates our space in two identical subspaces or cells, this is the topology of the "bicontinuous" cubic phases. The second corresponds to structures where a connected film defines an infinite number of infinitely long cells, this is the topology of the hexagonal and rectangular phases. The third topology corresponds to structures where a connected film defines an infinite number of finite cells, this is that of the nematic, "micellar" cubic and micellar phases.

The second result concerns the fact that any interfacial curvature can not be associated with any interfacial distance when an ideal structure is built in a curved space, they are indeed related by a specific relation in each topological class /31/. This set of relations is such that, when the frustration varies monotonously with the parameters of the phase diagram, the topologies must appear along a sequence similar to that observed in phase diagrams. For instance, when the water content is increased in a system with an amphiphile with one chain, a "bicontinuous" cubic and/or hexagonal phases and, finally, micellar phases are met after the lamellar phase.

The last result concerns the fact that the possible symmetries in each topological class compare well with those repertoried for ordered structures. The agreement is particularly striking in the case of cubic phases, "bicontinuous" /29,32/ and "micellar" /30/, presented above but is also valid for other structures $/ 28 /$. 


\section{CONCLUSION}

In this lecture we have tried to show that all structures formed by amphiphilic molecules in presence of water, around and above room temperature, can be analyzed as structures of films built by two facing liquid/1iquid interfaces. We have then examined these systems on this new basis, following a line very much inspired from that followed earlier to analyze the structures formed by atoms or molecules in classical condensed matter physics. We have tried first to establish a crystallography of films. More specifically, we have investigated the geometrical configurations permitting the films to conciliate constant interfacial distances and curvatures. The result of this first step is that these two constraints can be optimized only, through the introduction of disclinations. The ordered configurations obtained that way have topologies and symmetries corresponding to those of the liquid crystalline structures, their sequence when the curvature varies is also that commonly observed in the phase diagrams. This emphasizes the role of spatial constraints which, compared to those intervening in systems of atoms and molecules, are even more drastic here because of the dimensionality of the objects. This agreement, obtained without considering free energies, suggests also that their minima correspond to configurations closely related to those provided by crystallography. On this basis developments similar to those known for classical crystals are possible: relative stabilities of the configurations, fluctuations and defects, transformations of ordered structures into ordered or disordered ones. The latter point is particularly exciting, in the sense that some structural transformations imply dramatic topological changes of the structure of the $f i l m$, which do not exist in systems of atoms or molecules. These transformations might be driven by the appearance of defects in the structures described above. Such defects, or structural fluctuations, have been recently detected and their study is in progress /35/.

\section{REFERENCES}

/1/ Ekwal1, P., Adv. Liq. Cryst. Vol. 1, edited by Brown, G.H., (Academic Press, New York, 1975) p. 1.

/2/ Luzzati, V., in Biological Membranes, Vol. 1, edited by D. Chapman, (Academic Press, 1968) p. 71.

/3/ Skoulios, A., Ann. Phys. $\underline{3}$ (1978) 421.

/4/ Balmbra, R.R., Clunie, J.S. and Goodman J.F., Nature 222 (1969) 1159.

15/ Luzzati, V., Mariani, P. and Gulik-Krzywicki, T., in Physics of Amphiphilic. Layers, Springer Proceedings in Physics Vol. 21, edited by Langevin, D. and Meunier, J., (Springer Verlag, 1987), p. 131.

16/ Fontel1, K., Fox, K. and Hansson E., Mol. Cryst. Liq. Cryst. 1 (1985) 9.

/7/ Porte, G., Appe11, J. and Poggi, Y., J. Phys. Chem. 84 (1980) 3105. Candau, S., Hirsch, R. and Zana, R., in Physocs of Complex and Supramolecular Fluids, edited by Safran, S.A. and Clark, N.A., (Whiley Intersciences, 1987).

/8/ Rogers, J. and Winsor, P.A., J. Col1. Interface Sci. 30 (1969) 247.

/9/ Luzzati, V., Gulik-Krzywicki, T. and Tardieu, A., Nature 218 (1968) 1031.

/10/ Bangham, A.D., Adv. Lipid Res. 1 (1963) 65.

111/ Vanlerberghe, G., Nuovo Cimento $\underline{3}$ (1984) 219.

/12/ Hendrikx, Y. and Charvolin, J., J. de Physique 42 (1981) 1427.

/13/ Porte, G., Marignan, J., Bassereau, P. and May, R., J; de Physique 49 (1988) 511; Marignan, J., Appell, J., Bassereau, P., Delord, P., Larché, F. and Porte, G., Mol. Cryst. $152(1987) 153$.

/14/ Safinya, C., Roux, D., Smith, G.S., Sinha, S.K., Dimon, P., C1ark, N.A. and Bellocq, A.M., Phys. Rev. Lett. 57 (1986) 2513.

/15/ Bothorel, P., in Physics of Amphiphiles: Micelles, Vesicles and Microemulsions, edited by Degiorgio, V. and Corti, M. (North Holland 1985), p. 702 . 
/16/ Charvolin, J. and Hendricx, Y., in Nuclear Magnetic Resonance of Liquid Crystals, edited by J.W. Emsley, (Reidel Publishing Company 1985) p. 449.

/17/ Charvolin, J. and Rigny, P., J. Chem. Phys. $\underline{58}$ (1973) 3999.

/18/ Walderhaug, H., Soderman, O. and Stilbs. P., J. Phys. Chem. 88 (1984) 1655.

/19/ Kühner, W., Romme1, E., Noack, F. and Meier, P., Z. Naturforsch. 42a (1987) 127.

/20/ Charvolin, J., Manneville, P. and Deloche, B., Chem. Phys. Lett. 23 (1973) 345.

/21/ Sternin, E., Fine, B., Bloom, M., Tilcock, C., Wong, K. and Cullis, P., Biophy. J. 54 (1988) 689 .

122/ Charvolin, J., J. de Chimie Physique, 80 (1983) 15.

/23/ Jönsson, B. and Wennerström, H., J. de Phys. Chem. 91 (1987) 338, and references therein.

124/ Hendrikx, Y., Charvolin, J., Kekicheff, P. and Roth, M., Liq. Cryst. 2 (1987) 677.

/25/ Israelachvili, J.N., Intermolecular and surface forces, Academic Press (1985).

126/ Sadoc, J.F. and Mosseri, R., Pour la Science 87 (1987).

/27/ Harris, W.F., Sc. Am. 237 (1977) 130.

/28/ Sadoc, J.F. and Charvolin, J., J. de Physique 47 (1986) 683.

/29/ Charvolin, J, and Sadoc, J.F., J. de Physique 48 (1987) 1559.

/30/ Charvolin, J, and Sadoc, J.F., J. de Physique 49 (1988) 521.

/31/ Charvolin, J. and Sadoc, J.F., J. Phys. Chem. 92 (1988) 5787.

132/ Sadoc, J.F. and Charvolin, J., Acta Cryst. A45 (1989) 10.

133/ Schoen, A.H., NASA Tech. Note D-5541 (1970).

Hyde, S.T. and Andersson, S., Z: Krysta11. 168 (1984) 221.

Mackay, A.L., Nature 314 (1985) 604.

/34/ Rivier, N., Phil. Mag. B52 (1985) 795.

135/ Holmes, M.C. and Charvolin, J., J. Phys. Chem. 88 (1984) 810.

Kékicheff, P., Cabane, B. and Rawiso, M. , J. Phys. Lett. 45 (1984)L-813.

Hendrikx, Y., Charvolin, J., Kékicheff, P. and Roth, M., Liq. Cryst. 2 (1987) 677. Rançon, Y. and Charvolin, J., J. de Physique 48 (1987) 1067 and J. Phys. Chem. 92 (1988) $2646,6339$. 